\title{
The Simulation Analysis of Electromagnetic Repulsion Mechanism for
}

\section{High-voltage Current-Limiting Fuse}

\author{
Qiong $\mathrm{Wu}^{1,2}$, Shaohua $\mathrm{Ma}^{2}$,Zhiyuan $\mathrm{Cai}^{2}$ \\ ${ }^{1}$ Shen Yang university of technology, Shen Yang China \\ ${ }^{2}$ Shen Yang university, Shen Yang China \\ ${ }^{a}$ email, ${ }^{b}$ email
}

Keywords: Simulation, High-voltage, Electromagnetic Repulsion, Fuse

Abstract: The research content of this topic is based on Electromagnetic Repulsion Mechanism for High-voltage Current-limiting Fuse, according to the given parameters, and basing on the reasonable simplified, the topic establishes 2D finite element simulation model by using Ansoft Maxwell, and simulates the dynamic characteristics. The influence law of electromagnetic repulsion mechanism will be found out by simulating these influencing factors, such as repulsion mechanism, electromagnetic repulsion, and movement speed. Finally the topic concludes a general design guiding principle which strives to increase the speed of electric repulsion between contacts rapidly and improve the current-limiting ability of current-limiting fuse by producing short circuit current.

\section{Introduction}

The rapid vacuum switch based on electromagnetic repulsion mechanism has many characteristics, such as simple structure, fast switching, and smaller on-state loss comparing with high-power electronic devices. In recent years, with the research and development of short-circuit circuit-limiting device for the higher-requirement equipment in switch rapidity and reliability, the electromagnetic repulsion mechanism has been got attentions by many scholars from both at home and abroad.

\section{Validation Model of Repulsion Mechanism}

The validation model of electromagnetic repulsion mechanism is shown in figure 1 . The spring has already a certain amount of compression in the initial position, when the pulse current travels in separating-brake coil, the metal plate produces electromagnetic repulsion by induction eddy, under the function of electromagnetic repulsion, metal plate and metal connecting rod move downward together, and finally recovery to the initial state relying on the elastic of spring.

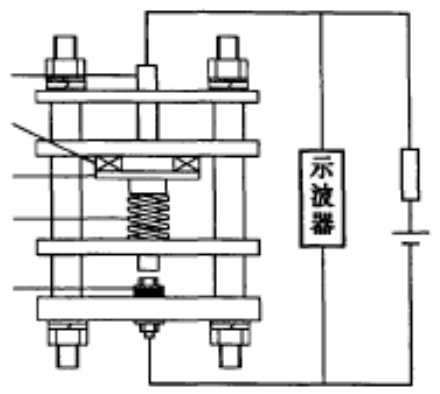

Fig.1 Validation Model of Electromagnetic Repulsion 


\section{Mechanism Simulation Analysis and Parameter Optimization}

In the case of structure size of known institutions and external circuit parameters, establishing the physical model in the Maxwell, and setting up external circuit model in Maxwell Circuit Editor from its own editor, and to solve the relevant parameters of the movement characteristics of electromagnetic repulsion mechanism.

3.1 Model and Parameter of Mechanism Simulation

Axial symmetric finite element model and the divided grid of electromagnetic repulsion mechanism are shown in figure 2.

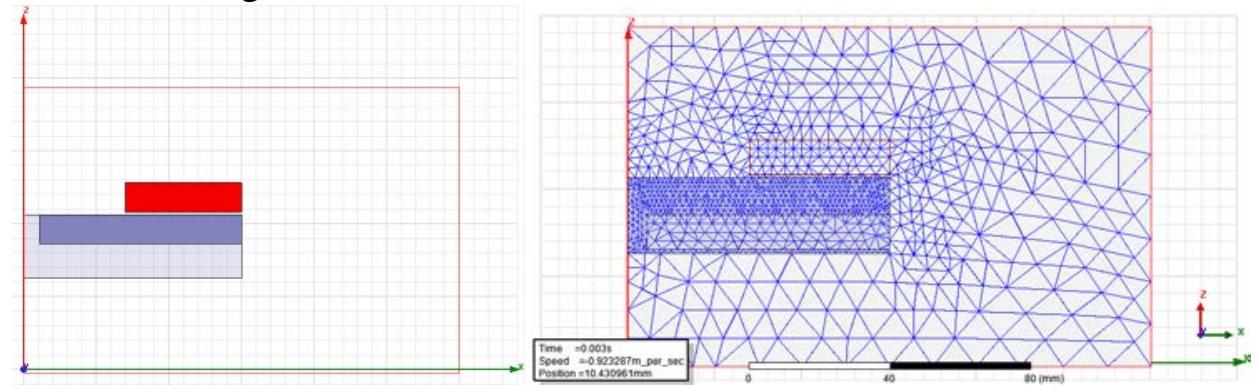

Fig. 2 Geometry Model and Divided Grid of Electromagnetic Repulsion Mechanism

According to the standard data given by manufacturer, the initial parameters of the simulation model as follows: coil material is red copper, coil inside radius $\mathrm{R} 0=35 \mathrm{~mm}$, outer radius $\mathrm{R}=75 \mathrm{~mm}$, turns number of coil is 28 , the material of metal plate is aluminum alloy, the radius metal plate is $75 \mathrm{~mm}$, the thickness of metal plate is $10 \mathrm{~mm}$, the initial clearance between the coil and the metal plate is $1 \mathrm{~mm}$, the quality of movable part (transmission rod, metal plate, dynamic contact) is $5 \mathrm{~kg}$, Moving distance is $16.9 \mathrm{~mm}$. 22 pieces of spring is in series connection, the compressed volume of separating-brake position is $3.5 \mathrm{~mm}$, pressure value is $3505 \mathrm{~N}$, reducing to 0 when $2.4 \mathrm{~mm}$, reversing to maximum $5500 \mathrm{~N}$ when $8.07 \mathrm{~mm}$, reducing to $3157 \mathrm{~N}$ when $11.5 \mathrm{~mm}$, the material properties of all parts of model is shown in table 1.

\begin{tabular}{c|c|c|c}
\hline Component & Material & Relative Permeability $\mu \mathrm{r}$ & $\begin{array}{c}\text { Conductivity } \gamma \\
(\mathrm{s} / \mathrm{m})\end{array}$ \\
\hline Coil & Red copper & 0.999991 & 56250000 \\
\hline $\begin{array}{c}\text { Metal Plate } \\
\text { Connecting Rod }\end{array}$ & $\begin{array}{c}\text { LY12 Aluminum } \\
\text { Alloy }\end{array}$ & 1.000021 & 22727000 \\
\hline
\end{tabular}

Ansoft transient field solver can couple with external circuit model set up by Maxwell Circuit Editor, shown in figure 3.

\subsection{Simulation analysis}

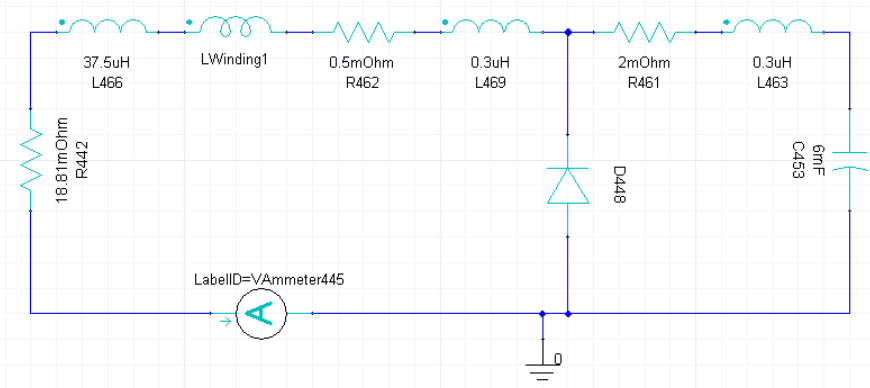

Fig.3 External Coupling Circuit Diagram

Setting the energy storage of capacitance is $1000 \mathrm{~V}$, simulation time is $3 \mathrm{~ms}$, and the simulation results are shown in figure 4. 


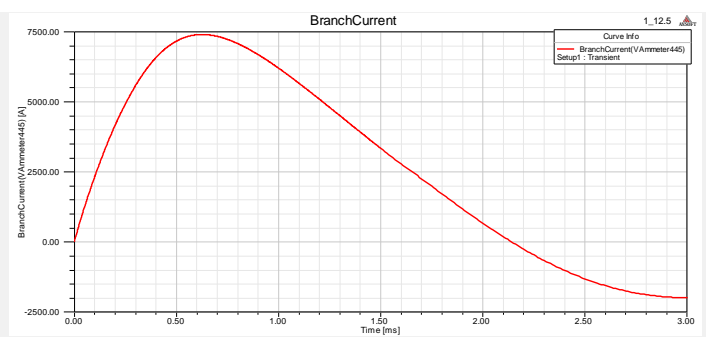

(a) Discharge Current

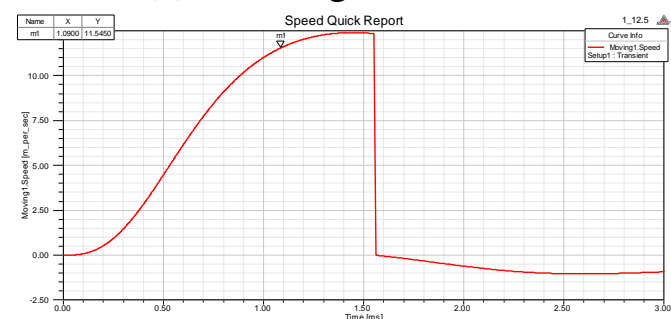

(c)Speed

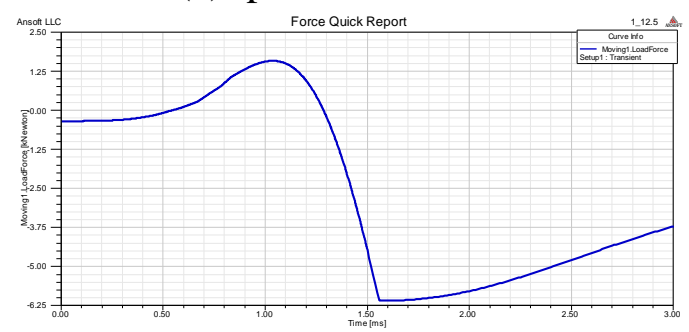

(e) Load Force

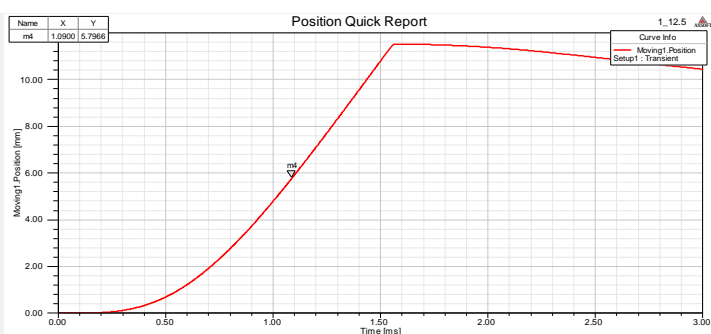

(b) Displacement-Time

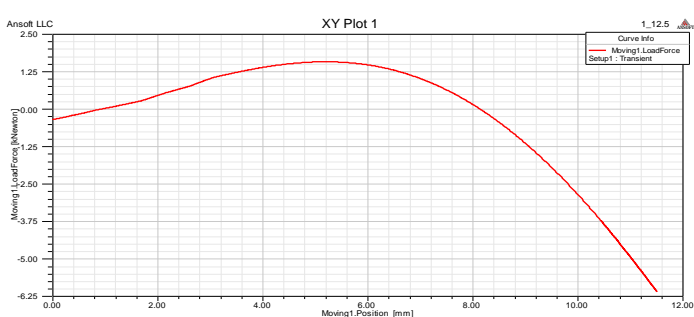

(d) Loading- Displacement

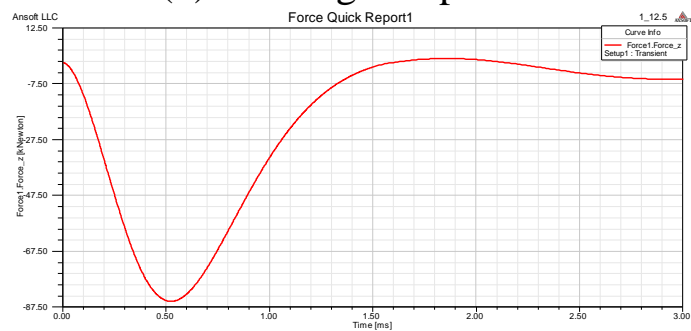

(f) Electromagnetic Repulsion

Fig.4 Simulation Results

Field distribution in each moment is shown in figure 5.

Magnetic line of force: 0.5, 1.0, 1.6

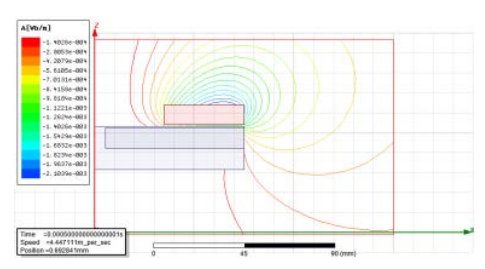

(a) $0.5 \mathrm{~ms}$ Magnetic Line of Force

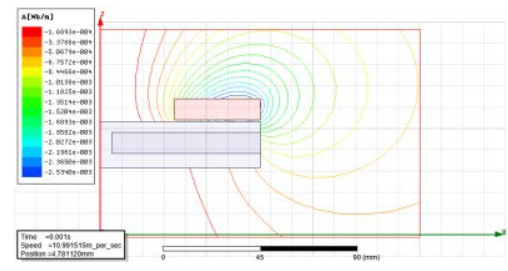

(b) 1ms Magnetic Line of Force

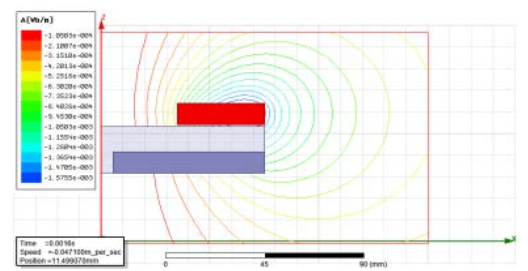

(c) 1.6ms Magnetic Line of Force

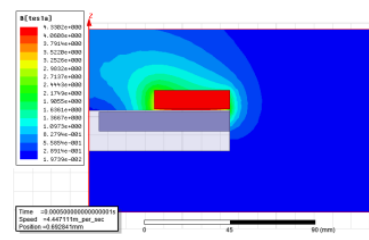

(d) $0.5 \mathrm{~ms}$ B Distribution

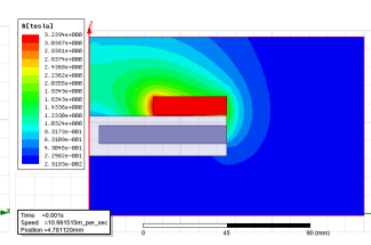

(e) 1ms B Distribution

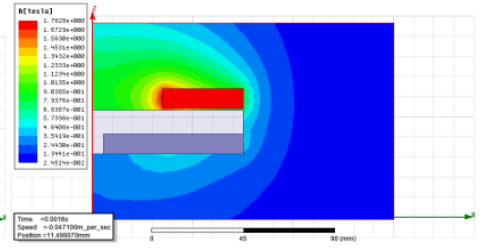

(f) $1.6 \mathrm{~ms} \mathrm{~B}$ Distribution

Fig.5 Diagram of Field Distribution in Each Moment

According to the simulation results, the maximum discharge current of the system is $7500 \mathrm{~A}$, and then declines exponentially. The full travel time of the mechanism is $1.55 \mathrm{~ms}$, The speed of movement is $7.4 \mathrm{~m} / \mathrm{s}$, the peak value of electromagnetic repulsion mechanism is 
82.5kn,and can achieve the purpose of opening and closing the test system quickly.

3.3 The Parameter Optimization and Simulation of Repulsion Mechanism

Basing on the simulation analysis of the model and basic structure parameters above, changing the main structure parameters of the system to simulate further, then the curve or data will be got by the influence of various parameters on the performance of electromagnetic repulsion mechanism.

1. The Influence of Radius, Material, and Thickness of Metal Plate to Mechanism

The influence to the peak value of electromagnetic repulsion by changing radius, material and thickness of metal plate is shown in table $1,2,3$.

\begin{tabular}{l|l|l|l|l|l|l|l}
\hline Radius of Metal Plate(mm) & 59.5 & 64.5 & 69.5 & 74.5 & 79.5 & 84.5 & 89.5 \\
\hline $\begin{array}{l}\text { Peak Value of Electromagnetic } \\
\text { Repulsion(kN) }\end{array}$ & 80 & 82.5 & 85.5 & 88.0 & 90 & 87.5 & 87.5 \\
\hline
\end{tabular}

Table 1. The Influence of Radius of Metal Plate to Peak Value of Electromagnetic Repulsion

\begin{tabular}{|c|c|c|}
\hline Material of Metal Plate(mm) & LY12 & Copper \\
\hline $\begin{array}{l}\text { Peak Value of Electromagnetic } \\
\text { Repulsion }(\mathrm{kN})\end{array}$ & 85.5 & 107.0 \\
\hline
\end{tabular}

Table 2. The Influence of Material of Metal Plate to Peak Value of Electromagnetic Repulsion

\begin{tabular}{l|l|l|l|l|l|l}
\hline Thickness of Metal Plate(mm) & 2.5 & 5 & 7.5 & 10 & 15 & 20 \\
\hline $\begin{array}{l}\text { Peak Value of Electromagnetic } \\
\text { Repulsion(kN) }\end{array}$ & 62.3 & 81.0 & 85.5 & 85.5 & 85.5 & 85.5 \\
\hline
\end{tabular}

Table 3. The Influence of Thickness of Metal Plate to Peak Value of Electromagnetic Repulsion

As shown in the table, the peak value of electromagnetic repulsion increases first then decreases, the maximum value will be got when the radius of metal plate is consistent with coil size. the repulsion can be increased obviously by changing the lower material of electrical resistivity; within a certain range, the thickness of metal plate is in same direction change to the peak value of electromagnetic repulsion, after more than a certain value, the electromagnetic repulsion will be almost the same.2. The Influence of Thickness, Material, and Clearance of Coil to Peak Value of Mechanism

\begin{tabular}{l|l|l|l|l|l}
\hline Thickness of Coil(mm) & 5 & 10 & 15 & 20 & 25 \\
\hline Peak Value of Electromagnetic Repulsion(kN) & 100 & 85.2 & 74.7 & 65.2 & 58 \\
\hline Time of Peak Value of Repulsion(ms) & 0.46 & 0.51 & 0.58 & 0.61 & 0.64 \\
\hline
\end{tabular}

Table 4. The Influence of Thickness of coil to The Peak Value and Occurrence Time of Mechanism

\begin{tabular}{l|l|l|l}
\hline Clearance of Coil $(\mathrm{mm})$ & 1 & 2 & 3 \\
\hline Peak Value of Electromagnetic Repulsion(kN) & 85.5 & 78.5 & 70.5 \\
\hline Time of Peak Value of Repulsion(ms) & 0.51 & 0.56 & 0.60 \\
\hline
\end{tabular}

Table 5. The Influence of Material of coil to The Peak Value of Electromagnetic Mechanism

As shown in the table, the peak value of electromagnetic repulsion reduces sharply by increasing the thickness of coil, and the peak time arrives ahead; The occurrence time of mechanism move backwards by widening the distance between metal plate and coil. 


\section{Conclusion}

This paper changes the parameters of system by analyzing the dynamic process of the simulation model of electromagnetic repulsion mechanism finite element. According to the simulation analysis, the influence of electromagnetic repulsion mechanism by the characteristic parameters has been got, and strives to increase the speed of electric repulsion between contacts rapidly when producing the short-circuit current, and then meets the design requirements of specific system.

\section{References}

[1] Dai Chao, Zhuang Jinwu, Yang Feng, et al. Pre-arcing Characteristics of Arctrigger for High Voltage Hybrid Current-Limiting Fuse [J]. High Voltage Engineering, 2010, 36(2): 350-355.

[2] Wang Chen, Zhuang Jinwu, Zhang Xiaofeng, et al. Principle and Test of a Novel Fault Current Limiter Based on IGBT for DC Power system [J].Transactions of China Electrotechnical Society, 2006, 21(9): 57-61.

[3] Toshie Takeuchi,Kenichi Koyama,Mitsuru Tsukima. Electromagnetic Analysis Coupled with Motion for High-Speed Circuit Breakers of Eddy Current Repulsion Using The Tableau Approach [J]. Electrical Engineering in Japan, 2005, 152(4):8-166

[4] Wang Zijian, He Junjia, Yin Xiaogen, et al. 10kV High Speed Vacuum Switch with Electromagnetic Repulsion Mechanism [J].Transactions of China Electrotechnical Society, 2009, 24(11): 68-75. 